\title{
Enteric Fever in Children of Nepal
}

\section{R. Leon Ochiai, MHS, International Vaccine Institute, Seoul, Korea.}

$\mathrm{W}^{\mathrm{s}}$

hen certain diseases catch the attention of the public, policymakers act rapidly. However, that is not the case for enteric fever. Ever since antibiotics became available and affordable, enteric fever became a disease of neighborhoods. In Nepal many people have individuals, including their friends and relatives have suffered from enteric fever at least once in their lifetime.

Studies published in this issue from the Civil Service Hospital and Birendra Hospital (Chhauni) in Kathmandu has provided important information on this "neighborhood disease" which his alarming in terms of its diagnosis, clinical management, and prevention considerations. During a one year period, these hospitals identified 46 culture positive typhoid cases. Although the number may seem very low in the highly populated, urban setting of Kathmandu, it is still noteworthy considering they were tertiary level hospitals and the data and were not from the cases identified in the community. If one can identify the health seeking behaviors of the population, it is likely that hundreds of cases are actually missed at hospital settings since febrile patients seek care typically at primary care clinics or go to a pharmacy for treatment.

In terms of diagnosis, the screening of febrile patients showed varying clinical presentations very rarely presenting specific typhoid signs or symptoms in these two studies. For example in medical textbooks classical typhoid patients are noted to present with rose spots, but from these studies, none came with such signs. With the absence of distinct clinical signs to diagnose typhoid fever, it is essential that proper laboratory tests be conducted. Widal tests may be commonly done, but they have been shown in many studies to lack adequate sensitivity and specificity. Also, there are reports of cross reactivity in these tests with other organisms. While blood culture tests remain the best option for identifying cases of typhoid fever, despite their low sensitivity (approximately $50 \%$ in optimal condition), they further require obtaining a large a volume of blood which often is a difficult choice for mothers of young children. Also, they require proper equipment and technical expertise for conducting clinical laboratory assessments. Even though such limitations exist for blood culture tests, they are important in their ability to isolate organisms and test for antibiotic sensitivity.

Findings of atibiotic susceptibility from the $S$. Typhi isolates of the two hospitals showed relatively low levels of resistance compared to neighboring South Asian countries. For example, studies from Pakistan consistently show very high levels of resistance to all first line antibiotics. Studies from various parts of India show relatively lower levels of resistance to first line antibiotics, though the majority are multi-drug resistant (resistant to all three first line antibiotics). Similar data have also been reported from Bangladesh. The current data from Civil Service Hospital and Birendra Hospital (Chhauni) are a good contrast to the data from Patan Hospital which from the late 1990s to early 2000s frequently reported multi-drug resistant $S$. Typhi. The important message is that antibiotic susceptibility can change over time and must be regularly tested to identify the most optimal clinical management for typhoid fever cases in Kathmandu and beyond.

Finally, it is important to disseminate the information that typhoid fever is a preventable disease. Provision of safe water, hygienic food, sanitary practices, and health education will likely reduce the cases of typhoid in the community. Especially, a thorough hand washing must be practiced as a general preventive measure. Individual efforts will continue to be important although there are challenges. The current state of the infrastructure of the Kathmandu Valley and city planning are reaching the limit with the high influx of people migrating to the Valley. Minimizing pollution, improving sanitation, managing water and electricity are among many other priorities for policymakers. While the government continues to work on numerous improvements in the society, paediatricians and public health experts will play an important role in advocating preventive health practices. Apart from seeking appropriate care at the early stages of diseases, vaccines can potentially be a major, cost effective preventive measure for society. There are two licensed typhoid vaccines internationally which have very good safety and efficacy profiles. One of them, the 
Vi polysaccharide vaccine, was in fact licensed based on a clinical trial conducted in the Kathmandu Valley in the 1980s. This vaccine, together with the other typhoid vaccine (Ty21a), are recommended for use by the World Health Organization for people living or visiting high risk areas, especially where antibiotic resistance is a concern, such as in Nepal.

The International Vaccine Institute (IVI) is currently working with the Government of Nepal to introduce the Vi polysaccharide typhoid vaccine through a school based immunization program. The initiative called VIVA (Vi-based Vaccines for Asia) is supporting the government in taking the lead role in a pilot program in Lalitpur District. The program will establish the logistic feasibility, safety and impact of the vaccine in order to support the government's efforts to establish National Guidelines for typhoid fever control. With the multidisciplinary, collaborative efforts of the government, healthcare practitioners, communities and individuals in typhoid case detection, management and prevention, I certainly hope that typhoid fever will no longer be a disease of neighborhoods and communities in the near future.

(R. Leon Ochiai, M.H.S., is Research Scientist at the International Vaccine Institute (IVI) in Seoul Korea. Mr. Ochiai received Master of Health Sciences (MHS) degree from the Johns Hopkins University School of Hygiene and Public Health, USA, and currently enrolled in Doctor of Philosophy (DPhil) program at the University of Oxford, UK. He has been involved in infectious disease epidemiology research in international settings, with more 10 years of field experience conducting onsite research activities in infectious diseases. Currently he is coordinating the VIVA (Vi-based Vaccine for Asia) Initiative, which aims to develop Vi-DT typhoid conjugate vaccine and to introduce Vi polysaccharide typhoid vaccine as a pilot public health program in Nepal and Pakistan.) 